\title{
RIGA IM POLNISCH-LITAUISCHEN STAAT (1581-1621)
}

\author{
von Anna Ziemlewska
}

Die Geschichte Rigas war bisher hauptsächlich ein Interessensgegenstand deutscher und deutsch-baltischer, in geringerem Maße russischer und sowjetischer, lettischer und schwedischer Forscher. Die deutsch-baltische und deutsche Geschichtsschreibung hat sich auf die Zeiträume der deutschen Dominanz in Livland (sog. Zeit der Selbständigkeit, Alt-Livland) konzentriert; daher hat sie der Entwicklung der Stadt und der sog. livländischen Konföderation (Gemeinschaft) im Mittelalter ${ }^{1}$ sowie dem Problem des Zerfalls der mittelalterlichen Territorialgewalten in Livland die größte Aufmerksamkeit gewidmet. ${ }^{2}$ In den Umkreis des Interesses deutscher Historiker gehört auch die Periode zwischen 1561 und 1581, als Riga den polnischen Königen den Huldigungseid verweigerte, die Union mit Litauen ablehnte und sich um die Wiederherstellung von Kontakten mit dem

\footnotetext{
' Friedrich BENNINGHOVEN, Rigas Entstehung und der frühhansische Kaufmann, Hamburg 1961: DERS.. Der Orden der Schwertbrüder. Fratres Milicie Christi de Livonia, Köln 1965; August von BUl.MERINCQ, Die Verfassung der Stadt Riga im ersten Jahrhundert der Stadt, Leipzig 1898; Friedrich Georg von BUNGE, Die Stadt Riga im dreizehnten und vierzehnten Jahrhundert Geschichte, Verfassung und Rechtzustand. Leipzig 1878; Manfred Hellmann, Der Deutschen Orden und die Stadt Riga, in: Stadt und Orden. Das Verhältnis des Deutschen Ordens zu den Städten in Livland, Preussen und im Deutschen Reich, hg. U. Arnold, Marburg 1993, S. 1-33: Gerd HolliHN, Die Stapel- und Gästepolitik Rigas in der Ordenzeit (1201-1562). Ein Beitrag zur Wirtschaftsgeschichte Rigas in der Hansezeit, in: HGbll. 1935, 60. S. 91-207; Constantin METTIG, Geschichte der Stadt Riga, Riga 1897; Ernst SeraPHIM, Geschichte von Livland, Bd. I (bis 1582), Gotha 1906.

${ }^{2}$ Gedruckte Quellen: Briefe und Urkunden zur Geschichte Livlands in den Jahren 1558-1562; hg. F. BienEMANN. Bd. I-V. Riga 1865-1881; Quellen zur Geschichte des Untergangs livländischer Selbstständigkeit, hg. K. SCHIRREN, Bd. 1-8, Reval 1861-1881; Neue Quellen zur Geschichte des Untergangs livländischer Selbstständigkeit, hg. K. SCHIRREN, Bd. 1-3. Reval 1883-86. Literatur zum Thema: Norbert ANGERMANN, Studien zur Livlandpolitik Ivan Groznyis. Marburg/Lahn 1972: Erich DONNERT, Der livländischer Ordensritterstaat und Russland. Der livländische Krieg und die Baltische Frage in der europäischen Politik 1558-1583, Berlin 1963. Dieses Thema hat auch schwedische und dänische Historiker interessiert u. a.: Sture ARNELL, Die Auflösung des livländischen Ordensstaates. Das schwedische Eingreifen und die Heirat Herzog Johans von Finnland, Lund 1937; Kund RASMUSSEN, Die livländische Krise 1554-1561, København 1973; Erik TiBERG, Zur Vorgeschichte des livländischen Krieges. Beziehungen zwischen Moskau und Litauen 1549-1562, Uppsala 1984.
} 
Reich und dem Haus Habsburg bemühte. ${ }^{3}$ Aus den Zeiten, während derer Riga innerhalb der Grenzen Polen-Litauens lag, griffen deutsche und deutsch-baltische Forscher meistens Probleme der (als polnisch bezeichneten) Gegenreformation in Livland und der Kalenderunruhen in Riga auf. ${ }^{4}$ Die polnische Geschichtsschreibung hat bisher ein geringes Interesse an der Geschichte Rigas und Livland gezeigt. ${ }^{5}$

In den Forschungen über die Geschichte Rigas bildet die sog. polnische Zeit (von dem 1581 zwischen einer Abordnung der Stadt und König Stephan Báthory geschlossenen Vertrag von Drohiczyn bis der Kapitulation Rigas vor den Truppen Gustav Adolfs im September 1621) bisher eine Lücke.

Das Problem der Zugehörigkeit Rigas zum polnisch-litauischen Staat ist sehr vielschichtig und kann unter verschiedenen Gesichtspunkten betrachtet werden: indem man den Schwerpunkt auf die inneren Angelegenheiten der Stadt legt (Sozialverhältnisse, Herrschafts- und Gildenorganisation, Aktivitäten von Handel und Handwerk) oder auf die Beziehungen der Stadt zur neuen Oberherrschaft und die Stellung der Stadt in Polen-Litauen (Rzeczpospolita).

Ziel meiner Forschungen ist es, die Art der Zugehörigkeit Rigas zu Polen-Litauen' und sein Handeln, seinen formalrechtlichen Status und seine tatsächliche Stellung im Staat aufzuzeigen, wie sie sich aus der alltäglichen Praxis, aus der Präsenz Rigas bei den Sejmsitzungen (Parlament) sowie dem Charakter der Verbindungen der Stadt mit den Herrschern und der Führungsschicht im Senat ergab.

\footnotetext{
${ }^{3}$ Friedrich BiEnemanN, Rigas Stellung bei der Auflösung des livländischen Ordenstaates, Russische Revue. Monatschrift für die Kunde Russlands, 11, 1877, S. 385-419; Wolfgang KüTTLER, Das Verhältnis der Stadt Riga zur Polen-Litauen in der Zeit der livländischen Krieges (1558-1583), in: Jahrbuch für Geschichte der UdSSR und der Volksdemokratischen Länder Europas, 10, 1967, S. 273-295; Wilhelm LENZ, Riga zwischen dem römischen Reich und Polen-Litauen in den Jahren 1558-1582, Marburg 1968; Herta von RAMM-HELMSING, Das staatsrechtliche Verhältnis der Stadt Riga zu Polen-Litauen im Zeitraum zwischen 1561-1581, JbbGOE 6, 1941, S. 171-200; Klaus-Dietrich STAEMMLER, Preussen und Livland in ihren Verhältnis zur Krone Polen 1561 bis 1586, Marburg/Lahn 1953.

${ }^{4}$ Die Besprechung der Quellen und der Literatur über die Gegenreformation und Kalenderunruhen siehe: Anna ZIEMLEWSKA, Rozruchy kalendarzowe w Rydze (1584-1589), ZapHist, 71, 2006, H. 1, S. 107-124.

${ }^{5}$ Roman CZAJA, Miasta inflanckie i estońskie w XIII-XVI wieku, in: Inflanty w średniowieczu. Władztwa zakonu krzyżackiego i biskupów. Toruń 2002; Władysław CZAPLIŃSKI. Stanowisko państw skandynawskich wobec sprawy inflanckiej w latach 1558-1561, ZapHist, 28, 1963, H. 3, S. 379-406; Stanislaw KARwowSKI, Wcielenie Inflant do Litwy i Polski 1558-1561, Poznań 1873; Abdon KŁodZIŃSKI, Stosunki Polski i Litwy z Inflantami przed zatargiem z 1556/67, Kwartalnik Historyczny, 22, 1908. S. 344-391; Jan KosTRZAK, Narodziny ogólnoinflanckich zgromadzeń stanowych od poł. XIII do poł. XV w. (Roczniki TNT, Jg. 82, H. I), Warszawa 1985; Polska a Inflanty (Pamiętnik Instytutu Bałtyckiego 39, hg. v. J. BoROWIK), Gdynia 1939.
} 
Der Angriff der Truppen Ivan Groznys auf Livland im Jahr 1557 führte zum Zerfall der bisherigen Territorialherrschaften. Dänemark, Schweden, Litauen und Polen begannen, um deren Lande zu rivalisieren. 1561 begaben sich in Wilna die bisherigen Herren Rigas, der Rigaer Erzbischof Wilhelm von Hohenzollern und der letzte livländische Ordensmeister, Gotthard Kettler, unter die Obhut des polnischen Königs Sigismund August. Riga, nicht unmittelbar von den russischen Truppen bedroht, folgte dem Erzbischof und dem Ordensmeister nicht und wollte sich Sigismund August nicht unterwerfen. Zwar legte der Rat der Stadt nach viele Monate dauernden Verhandlungen mit Nikolaus Radziwill, nach der Bestätigung ihrer Privilegien und der Konfessionsfreiheit sowie der Garantie des Anschlusses an die Krone in der sog. Cautio Radziwillana gegenüber den Vertretern des Königs einen Huldigungseid ab, doch als die polnisch-litauische Seite nicht in der Lage war, alle Bedingungen zu erfüllen (es ging hauptsächlich um eine Zuordnung der Stadt zu Polen und nicht zu Litauen), verweigerte die Stadt den Gehorsam und akzeptierte weder die Oberherrschaft Litauens noch die Formel einer unmittelbaren Unterordnung unter den König. Sie handelte so selbst um den Preis politischer Isolierung sowie militärischer und wirtschaftlicher Repressionen.

Es fällt schwer, den formalrechtlichen Status der Stadt in den sechziger und siebziger Jahren des 16. Jahrhunderts genau zu umschreiben. Obwohl Erzbischof und Orden formell ihre Rechte an Riga an Sigismund August übergeben und eine litauische Besatzung das Rigaer Schloss und die Festung Dünamünde besetzt hatten, hatte der König keine tatsächliche Macht über die Stadt. Das reiche Riga, das den Handel im Einzugsbereich der Düna monopolisiert und ganze Scharen litauischer Adliger von seinen Krediten abhängig gemacht hatte, betrieb über 20 Jahre hinweg eine unabhängige Politik: es erkannte die Herrschaft der Administratoren Livlands, Kettler und Chodkiewicz, über die Stadt nicht an, es zerstörte die von den königlichen Truppen errichteten und die Schifffahrt behindernden Befestigungsbauten, sog. Blockhäuser, und besetzte 1563 eigenmächtig die Besitztümer des verstorbenen Erzbischofs Wilhelm. Sigismund August vermied einen offenen Konflikt mit Riga, selbst nach dessen entschiedenem Widerspruch gegen die Union mit Litauen und der Kontaktaufnahme mit dem Reich und Herzog Magnus von Dänemark. In derselben Zeit betrieb Sigismund August eine entschiedene Politik gegenüber Danzig, um die Unabhängigkeit der Stadt zu beseitigen. Die Politik des Königs gegenüber Danzig entsprach den Interessen der polnischen Schlachta (des polnischen Adels) und hatte deswegen die Unterstützung des Sejm und der Machtelite des Landes. Anders sah es im Fall Rigas aus. Die polnische Schlachta wollte sich nicht in den Angelegenheiten des fernen Livland 
engagieren. Die Unterstützung der am Zugang zum Rigaer Hafen und an einer Ausweitung des Exports von Getreide, Hanf und Waldprodukten interessierten litauischen Schlachta erwies sich als nicht ausreichend. Wichtiger als eine Bestimmung des Status' der Stadt nach 1561 ist es, eine Antwort auf die Frage zu finden, warum die Stadt die Oberherrschaft Sigismund Augusts und Litauens nicht annehmen wollte. Um den Standpunkt der Stadt zu verstehen, muss die innere Situation in Riga analysiert werden.

Die im 16. Jahrhundert in Riga auftretenden gesellschaftlichen Veränderungen führten zu einem Auseinanderdriften der Machtelite aus Großkaufleuten, Gutspächtern und Kreditgebern, die viel Geld in Land investierten, wie Angehörige der Schlachta lebten und eine Vereinigung mit dem litauischen Hinterland wollten, sowie der bürgerlichen Opposition, zu der aktive Kaufleute, Krämer und Handwerker gehörten. Sie fürchteten eine Stärkung der Position des Rates, den Verlust von Privilegien und die Unterbrechung alter Handelsverbindungen. Sie waren nicht so sehr gegen eine Oberherrschaft Sigismund Augusts und Litauens, als vielmehr gegen jegliche Art starker Übermacht. So lange, wie der Rat die Unterstützung der Gilden für Steuerbeschlüsse zu Militärzwecken oder zum Ausbau der Befestigungsanlagen benötigte, so lange betrieb Riga eine konsequent separatistische Politik und zielte auf die Wiederherstellung der Verbindungen zum fernen und, was damit einherging, für die Stadt ungefährlichen Reich. ${ }^{6}$

Ein weiterer Angriff Ivan Groznys auf Livland im Jahr 1577 zwang die Stadt, ihre Haltung zu überdenken und eine Annäherung an Polen und Litauen zu suchen. Trotz der realen Gefahr wurden die Bedingungen für eine Unterordnung der Stadt unter Polen-Litauen erst nach langen, mehrfach abgebrochenen Verhandlungen und Diskussionen über den Umfang der königlichen Herrschaft in der Stadt, die Aufteilung der Zolleinkünfte, über die Besitzungen des Erzbischofs und endlich die Konfessionsfreiheiten ausgearbeitet. Zum Schluss erließ Stephan Báthory 1581 in Drohiczyn das sog. Privilegium Stephaneum für die Stadt und die Rigaer Abordnung leistete endlich den Huldigungseid. ${ }^{7}$ Der König bestätigte die alten Handelsprivilegien der Stadt. Sie musste nur die Einnahmen aus dem nach Danziger Vorbild eingeführten Pfahlzoll (sog. Portorium) mit dem König teilen ( $1 / 3$ für die Stadt). Der Rat behielt große Selbständigkeit. Báthory behielt sich lediglich das Genehmigungsrecht für Verfassungsänderungen

\footnotetext{
${ }^{6}$ LENZ, Riga (wie Anm 3).

${ }^{7}$ Der Vertrag von Drohiczyn wurde von der Opposition als Verrat bewertet. Zwei Mitglieder der Abordnung wurden einige Jahre später verurteilt und hingerichtet.
} 
und die jährliche Wahl des Burggrafen vor, der der Vertreter des Herrschers in Riga war.

Báthorys Privileg hatte grundlegende Bedeutung für den Status Rigas im Rahmen des polnisch-litauischen Staats, regelte jedoch nicht alle mit der Existenz der Stadt unter der neuen Obrigkeit zusammenhängenden Fragen. Religionsangelegenheiten, Fragen des Eigentums an den ehemaligen Gütern des Kapitels und des Erzbischofs sowie das Problem des Handels der litauischen Schlachta, der freien Schifffahrt usw. wurden durch gesonderte Mandate geregelt, die 1582 während des Aufenthaltes Báthorys in Riga erlassen wurden. ${ }^{8}$

Der König sicherte den Protestanten die Konfessionsfreiheit zu und verzichtete auf die ehemaligen Güter der Rigaer Erzbischöfe, tat das aber im Tausch gegen die Übergabe zweier Kirchen für katholische Gottesdienste (St. Jakobi und St. Maria Magdalenen mitsamt den Klostergebäuden). Rigas Machtelite ließ sich darauf ein, die Opposition aber kämpfte um die alleinige Gültigkeit der Augsburgischen Konfession und den Erhalt der Kirchen, berief sich auf die von Sigismund August erhaltenen Privilegien und rief in Erinnerung, dass die Unterwerfung unter Polen-Litauen freiwillig erfolgt sei. Es gelang ihr jedoch nicht, die Haltung des Königs zu ändern.

Der siegreiche Herrscher führte, anders als Sigismund August, die neuen Regelungen in Livland und Riga aus einer Position der Stärke ein (ius gladii). Er setzte die Rückkehr des Katholizismus nach Livland durch, die Wiederherstellung der kirchlichen Hierarchie mit dem Bistum in Wenden, die Rückgabe einiger Kirchen und Landgüter und schließlich die Errichtung von Jesuitenkollegs. Die Unterstützung der Gegenreformation in Livland war für Báthory ein politischer Vorgang, ein Element des Prozesses der Integration dieses Gebiets in den polnisch-litauischen Staat. ${ }^{9}$

Ein Element des Integrationsprozesses in den Ländern Polen-Litauens bildete der gregorianische Kalender. Die durch die Einführung dieses gregorianischen Kalenders in Riga von 1584 hervorgerufenen Kalenderunruhen (bekannt auch aus anderen Städten und Ländern) verwandelten sich in Riga in einen fünfjährigen Zeitraum der Wirren und Kämpfe. Die

\footnotetext{
${ }^{8}$ Druck der Privilegien und der Mandate Bathorys für die Stadt: M. DoGiEl, Codex Diplomaticus Regni Poloniae et Magni Ducatus Lithuaniae, Bd. 5 in quo ut universae Livoniae ita speciatim Curlandiac et Semigaliae ducatum res continentur, Wilno 1759, S. 308-314.

"In der deutschen und deutsch-baltischen Geschichtsschreibung wurde ein schreckliches Bild der ,polnischen Gegenreformation“ gezeichnet; die neuesten Arbeiten lettischer Historiker u. a. Gvido STRAUBE, Die „polnische Gegenreformation“ in Livland - Ziele und Realitäten, in: Prusy i Inflanty między średniowieczem a nowożytnością. Państwo-spoleczeństwo-kultura, hg. B. Dybaś, D. Makiłła, Toruń 2003, S. 117-123, zeigen, dass das polnische und jesuitische Handeln sehr geringe Wirkungen hatte.
} 
Opposition mit Martin Giese an der Spitze übernahm faktisch die Macht in der Stadt, führte nicht nur die alte Zeitrechnung wieder ein, sondern verurteilte auch zwei Unterzeichner des Vertrags von Drohiczyn zum Tode (andere Anhänger der polnischen Herrschaft fanden Schutz in der Burg oder am königlichen Hof), verjagte die Jesuiten, nahm den Katholiken die Kirchen (St. Jakobi und St. Maria Magdalenen) weg und ignorierte die Befehle des Königs und seines Statthalters. Der Tod Báthorys 1586 und das lange Interregnum, die Doppelwahl und die inneren Konflikte in Polen-Litauen erlaubten es der bürgerlichen Opposition, sich bis 1589 an der Macht zu halten, als königliche Kommissare die Herrschaft des Rates der Stadt wiederherstellten und den Treueid der Stadt entgegennahmen. ${ }^{10}$ Für deutsch-baltische Historiker waren diese Ereignisse ein Symbol des Widerstands der deutschen protestantischen Bevölkerung gegen die von der polnischen (lies: fremden) Obrigkeit aufgenötigte Gegenreformation. Für einige Forscher waren die Unruhen ein religiöser, für andere ein gesellschaftlicher oder politischer Konflikt, ein Höhepunkt der Kämpfe einer bürgerlichen Opposition mit der Machtelite. " Für Forschungen über Riga im Polen-Litauen ist es das Wichtigste festzulegen, welchen Einfluss der Konflikt in Riga auf die Beziehungen zur Zentralgewalt, zum König und zum Statthalter des Königs in Livland hatte.

Äußerst vielschichtig sind die Aufgabe und die Versuchung, alle Aspekte des Lebens und Treibens Rigas, vor allem die Rolle und Präsenz der Stadt im politischen Leben Polen-Litauens unter der Regierung Sigismunds III. darzustellen und die Art der Kontakte der Stadt mit dem Hof, mit der polnisch-litauischen Verwaltung und dem Militär zu bestimmen. Es ist wichtig zu zeigen: Wie hat sich die Stadt auf die politischen Gepflogenheiten in Polen-Litauen eingestellt? Wie war sie auf den Sejmsitzungen vertreten? Wie gestaltete sie die Beziehungen zu König und Würdenträgern, zur livländischen und litauischen Schlachta sowie zu den lokalen Amtsträgern? ${ }^{12}$

${ }^{10}$ Wolfgang KüTTLER, Patriziat, Bürgeropposition und Volksbewegung in Riga in der zweiten Hälfte des 16 Jahrhunderts, Leipzig 1966 (nicht gedruckte Dissertation), S. 281-334.

"ZIEMLEWSKA, Rozruchy (wie Anm. 4), S. 107-124.

${ }^{12}$ Die Zugehörigkeit zur „Rzeczpospolita bedeutete nicht nur Kontakte mit dem Hof oder mit der polnisch-litauschen Verwaltung. Ein Bild von der polnisch-litauischen Herrschaft in Livland und den alltäglichen Problemen der Stadt ergibt sich erst, wenn man die Beziehungen zwischen der Stadt und den benachbarten Festungen untersucht. Zwischen der Stadt und der Burg brachen immer wieder Konflikte u. a. um die Größe der Vorburg und die dort wohnenden Handwerker. das Bierbrauen, den Zustand und die Belieferung der Burg und wegen Überfällen der Besatzung auf Rigaer u. ä. auf; mit der Festung Dünamünde stritt Riga um die freie Schifffahrt auf der Düna, den Leuchtturm, die Lotsen und den Fischfang; die Kommandanten, wie Jan Ostrowski oder später Wolmar Farensbach, hielt man für Feinde der Stadt. Latvijas Valst Vēstures Arhīvs (LVVA Lettisches Historisches Staatsarchiv), f. 673-1 
Das erhaltene reiche Quellenmaterial, wie Instruktionen für Sejmsitzungen und Berichte aus ihnen, Protokolle und Beschlüsse (Konstitutionen) des Sejm, erlaubt es zu verfolgen, wie die Rigaer Abgeordneten Angelegenheiten wie die Erringung neuer Privilegien und Darlehen, die Vertreibung der Jesuiten, die Bestätigung des freien Handels und freier Schifffahrt u. ä. erledigten. ${ }^{13}$

Die Korrespondenz Rigas mit Sigismund III. und hochgestellten Personen (Würdenträgern), königliche Mandate und Urteile aus Gerichtsverhandlungen zeigen die Beziehungen mit dem Hof und dem König selbst. ${ }^{14}$ Sigismund III. Vasa, der nach dem Tod seines Vaters um den Erhalt und später die Wiedererringung des schwedischen Thrones kämpfte, schenkte den Ostseestädten besondere Beachtung, die Schiffe, Militär und Waffen liefern, Geld verleihen und im Falle eines Angriffs Schwedens einen Schutz für die polnischen Besatzungen in Livland und Königlich Preußen darstellen konnten. Der Anfang der Herrschaft Sigismunds III. ließ gute Beziehungen zwischen dem Herrscher und der Stadt nicht erwarten. Das von Martin Giese regierte Riga unterstützte bei der Wahl des Jahres 1587 die habsburgische Kandidatur und wollte die Wahl Sigismunds nicht anerkennen. Erst im Jahr 1589 und nach dem Einschreiten königlicher Kommissare huldigte die Stadt dem Herrscher. Sigismund III. forderte konsequent die Rückgabe der Kirche St. Jakobi an die Katholiken ein und unterstützte die Aktivität des Jesuitenkollegs in Riga. In anderen Fällen kam er der Stadt jedoch freundlich entgegen. Er bestätigte ihre früheren Privilegien und entsandte Kommissare zur Verhandlung von Streitigkeiten der Stadt mit der Burg, mit der Festung Dünamünde und mit der livländischen Schlachta. Er unterstützte eine Reinigungsaktion auf der Düna, verbot das Bierbrauen in der Nähe der Stadt, die Benutzung von Nebenhäfen und der Schlachta das Handeltreiben, befahl die Beendigung der Eigenmächtigkeit von polnisch-litauischen Soldaten in Livland u. ä. Die Beziehungen des Königs zur Stadt verdichteten sich noch während des Krieges in Livland (1600-1621)..$^{15}$

Riga war der Schlüssel zu Livland, zur Kontrolle über die Düna, über sein Hinterland und die Transitwege nach Osten (Kampf um Livland bedeutet Kampf um Riga). Im Gegensatz zur livländischen Schlachta unter-

Rĩgas Rātes Ārējais Arhīvs (Äuesseres Ratsarchiv). Nr. 1007-1015 Subcastrensia: Nr. 93-105 Dunamundensia.

${ }^{13}$ LVVA, f. 673-3, Rīgas Rātes Ārējais Arhīvs, Kaste 10-17.

${ }^{14}$ LVVA, f. 673-1. Nr 1219 Mandatorum Regni Poloniae Extracta 1570-1630; Nr 1220 Acta Commissionis generalis Poloniae; Biblioteka Kórnicka Polskiej Akademii Nauk, Handschriften: Nr 293, Nr 294, Nr 312.

15 Über den Krieg in Livland siehe: Stanisław HERBST, Wojna inflancka 1600-1602, Warszawa 1938; Henryk WISNER, Kircholm 1605, Warszawa 2005. 
stützte die Stadt nicht Karl IX. und verblieb sogar bis 1621 unter der Obrigkeit Polen-Litauens. Das Wort „verblieb“ legt eine passive Haltung nahe, Riga dagegen nahm aktiv am Krieg teil: Die Stadt belieferte das Militär mit Lebensmitteln, Waffen und Munition, gewährte Darlehen für Sold und neue Aushebungen, informierte über die Erfolge der schwedischen Truppen zu Lande und zur See, unterhielt ein eigenes Kontingent, wehrte mehrfach Angriffe der Schweden ab (1601, 1605), befreite mit eigenen Kräften und besetzte mit einer eigenen Besatzung die Festung Dünamünde und setzte die Rigaer Burg in Stand usw. ${ }^{16}$

Berücksichtigt man die Umstände der Unterwerfung Rigas unter PolenLitauen, den Widerstand der Bürgerschaft gegen die polnische Machtelite und die Gegenreformation sowie die Konflikte mit der Burg und der Festung Dünamünde, kann eine solche Haltung erstaunen. Eindeutig lohnte es sich für Riga und seine Einwohner, bei Polen-Litauen zu verbleiben. Die erzielten Einnahmen und Umsätze (bzw. die Hoffnung auf eine Rückkehr zu dem Niveau, das am Ende des 16. Jahrhunderts bestand) überwogen anscheinend die Mühen und Kosten der Kriegsführung und Verluste, die sich aus der Hafenblockade, der Zerstörung des livländischen Hinterlandes, der Unterbrechung der Handelswege und dem Raub von städtischen Gütern durch schwedische, aber auch polnische und litauische Truppen ergaben. ${ }^{17}$ Königliche Kommissare, König und Sejm lobten die Haltung der Stadt. Das spiegelte sich in Sejmbeschlüssen und in der Korrespondenz. Zur Deckung der Kosten für den Unterhalt des Militärs und die Ausbesserung von Kriegsschäden erhielt Riga die Hälfte der Einkünfte aus dem Pfahlzoll (Portorium). Es konnte auch auf das Wohlwollen des Königs bei Entscheidungen über die Auseinandersetzungen der Stadt mit den Jesuiten, königlichen Beamten, der livländischen Schlachta und den kurländischen Herzögen zählen. ${ }^{18}$ Doch im September 1621 musste sich Riga nach mehrwöchiger Belagerung Gustav Adolf ergeben. Damit endete die polnische Herrschaft für die Stadt definitiv.

Riga war nur 40 Jahre unter polnisch-litauischer Herrschaft, zu kurz um eine wichtige Rolle in der Geschichte von Polen-Litauen zu spielen, obwohl es dazu große Chancen hatte. Riga gehörte zu der kleinen Gruppe von Städten, die in Polen-Litauen eine relativ große Selbständigkeit hat-

\footnotetext{
${ }^{16}$ Eriks JEKABSONS. Miasto Ryga i twierdza Dźwinoujście podczas wojny polsko-szwedzkiej 1600-1621, Zeszyt Naukowy Muzeum Wojska w Białymstoku, 12, 1998, S. 19-36; ZIEMLEWSKA, Trwanie przy Rzeczypospolitej. Postawa Rygi wobec państwa polsko-litewskiego na przełomie XVI i XVII wieku (im Druck).

${ }^{17}$ Rigas Handel siehe: Wasilij DoRoszenKo, Eksport Rygi na Zachód w okresie przynaleźności do Rzeczypospolitej 1562-1620, ZapHist, 31, 1966, H. 1. S. 7-44; DERS., Riga und sein Hinterland im 17 Jahrhundert, Hansische Studien 4, Weimar 1979, s. 155-172.

${ }^{18}$ LVVA, f. 673-3, Rīgas Rātes Ārẽjais Arhīvs, Kaste 14-17.
} 
ten. Die Analyse der königlichen Privilegien und Mandate für die Stadt erlaubt es festzustellen, dass Riga einen ähnlichen rechtlichen Status wie die preußischen Städte erhielt. Das Muster war hier zweifellos Danzig, mit dem die Rigaer Machtelite oft in Kontakt traten und bei dem sie um Rat baten. Der Prozess der Unterordnung der Stadt unter Polen-Litauen verlief jedoch unter anderen Umständen und in einer entscheidend ungünstigeren Zeit (Zeitraum einer Dominanz der Schlachta und des Abdrängens der Städte an den Rand des politischen Lebens). Den polnischen und litauischen Städten waren in der Frühen Neuzeit die politischen Rechte genommen worden, nur einige von ihnen, $u$. a. Riga, schickten ihre Gesandten zu Sejmsitzungen. Schon die Teilnahme Rigas am politischen Leben zeigt seinen speziellen Status in Polen-Litauen.

Das reiche Riga monopolisierte den Handel im Dünagebiet (Nordlitauen und Weißrussland) und hatte eine sehr große Anzahl litauischer Adliger von seinen Krediten abhängig gemacht. Es war neben Danzig der größte Hafen in Polen-Litauen und während des Krieges mit Schweden die größte und treueste Festung in Livland. Polen-Litauen hat im Jahre 1621 zu wenig getan, um die Stadt unter seiner Herrschaft zu halten. 